\title{
Predicting treatment response using pharmacy register in migraine
}

\author{
Thomas Folkmann Hansen ${ }^{1,2^{*}}$ (D), Mona Ameri Chalmer ${ }^{1}$, Thilde Marie Haspang ${ }^{1,3}$, Lisette Kogelman ${ }^{1}$ and \\ Jes Olesen ${ }^{1}$
}

\begin{abstract}
Background: Precision medicine may offer new strategies to treat migraine, and access to existing large cohorts may be a key resource to increase statistical power. Treatment response data is not routinely collected for large cohorts; however, such information could be extracted from pharmacy databases. Using a clinical migraine sample with treatment effect data, we assessed whether treatment response can be predicted based on the number of drug purchases.
\end{abstract}

Methods: A clinical cohort including 1913 migraineurs were interviewed using a semi-structured interview to retrieve treatment response data for acute and prophylactic migraine drugs. The purchase history was obtained from the Danish national pharmacy database. We assessed whether number of purchases at different thresholds could predict the specificity and sensitivity of treatment response.

Results: Purchase history of drugs was significantly associated with treatment response. For triptan treatment the specificity and sensitivity were above $80 \%$ for individuals with at least ten purchases. For prophylactic treatment (beta-blockers, angiotensin II antagonists or antiepileptic) we observed a sensitivity and specificity above $80 \%$ and $50 \%$ for individuals purchasing any prophylactic drug at least four times. In the Danish pharmacy database, $73 \%$ of the migraine patients have purchased at least ten triptans, while 55-63\% have purchased at least one of the four prophylactic drugs.

Conclusion: Pharmacy databases are a valid source for identification of treatment response. Specifically for migraine drugs, we conclude that ten purchases of triptans or four purchases of prophylactic drugs are sufficient to predict a positive treatment response. Precision medicine may be accelerated with the use of pharmacy databases.

Keywords: Pharmacy database, Treatment response, Treatment predictors, Migraine

\section{Background}

The success of precision medicine depends on the ability to identify patient groups with a specific response to a drug. The predictive power to classify patients depends on the quality and size of the initial cohort used to build the models [1]. Retrieving treatment response data for a large cohort is resource intensive and can take a long time. Identification of treatment response markers, e.g. in existing pharmacological databases that are easily

\footnotetext{
* Correspondence: thomas.hansen@regionh.dk

1Danish Headache Centre, Department of Neurological department, Copenhagen University Hospital, Nordreringvej 69, DK-2600 Glostrup, Denmark

${ }^{2}$ Novo Nordisk Foundation Center for Protein Research, Faculty of Health and Medical Sciences, University of Copenhagen, Copenhagen, Denmark

Full list of author information is available at the end of the article
}

accessible, is an alternative and simple strategy for assessing large cohorts. Although several countries maintain registry databases with information on individual medication use [2], this approach has never been tested. Migraine is an ideal condition to test the accuracy of prediction of treatment response from pharmacological databases. Migraine affects $15-20 \%$ of the population [3] and both acute and prophylactic treatments are available [4]. Triptans are migraine-specific acute drugs with no effect on peripheral pain [5] and are reported to be effective in $60-70 \%$ of treated migraine patients [6]. There are several (non-specific) prophylactic drugs for migraine available and the current choice of prophylactic treatment is made by trial and error $[4,7]$. 
In Denmark, the national pharmacy database holds individual-level data on all prescriptions and subsequent purchases of drugs [8]. In a large clinical sample of migraine patients, we have collected information regarding migraine treatment response for both acute and prophylactic drugs [9]. We hypothesized that drug purchases can predict treatment response. To test this hypothesis, we combined the Danish pharmacy database with our clinical migraine sample and provide an estimate of the sensitivity and specificity to predict treatment response.

\section{Methods}

Patients were recruited as part of the migraine genetic cohort at the Danish Headache Centre (tertiary headache referral centre) from 2010 to 2016. All patients were interviewed by medical doctors or senior medical students specifically trained in using a semi-structured interview to diagnose headache according to the International Classification of Headache Disorders (ICHD) [10]. A total of 1913 migraine patients with or without typical aura answered questions regarding medication use and treatment response to relevant headache pharmacological treatments including acute treatment (following categories: triptans (general, non-specific), ergotamine, non-migraine specific analgesics) and prophylactic treatment (following categories: Beta-blockers, Angiotensin II antagonists, Antiepileptics, ACE-inhibitors, and Anti-depressives). Acute treatment effect was considered positive if the patient reported at least $50 \%$ pain reduction within two hours of taking the drug. Prophylactic treatment was considered effective if the patient reported a reduction of at least $50 \%$ in the number of migraine attacks with three months of drug use. Patients who did not remember or had not tried the medication in question were registered as missing data.

The Danish medical prescription register is a national database in which all purchases of drugs prescribed by a medical doctor have been registered since 1994; data from 1994 until 2016 was included in the analysis. The register is primarily used for socioeconomic evaluation of medication use in Denmark. Prescription data for headache treatments often used in Denmark (Table 1) were merged at Denmark Statistics where study participants were fully anonymized. In Denmark, it is possible to buy non-migraine specific analgesics over the counter (OTC) and it is not possible to retrieve information about the purchase of non-migraine specific analgesics.

\section{Statistics}

We used R version 3.4.1 in RStudio version 2.1 for statistical analysis, with the $\mathrm{R}$ packages sas7bdat, ggplot2, caret, and ROCR. We created a confusion matrix for treatments reported with the effective measure in more than $5 \%(n=$
Table 1 Drugs assessed in pharmacy database

\begin{tabular}{|c|c|c|}
\hline & Drug & ATC Code \\
\hline \multirow[t]{17}{*}{ Acute } & Triptans & \\
\hline & Sumatriptan & N02CC01 \\
\hline & Zolmitriptan & $\mathrm{N} 02 \mathrm{CCO} 3$ \\
\hline & Naratriptan & $\mathrm{N} 02 \mathrm{CCO} 2$ \\
\hline & Rizatriptan & $\mathrm{N} 02 \mathrm{CCO} 4$ \\
\hline & Almotriptan & $\mathrm{N} 02 \mathrm{CC} 05$ \\
\hline & Eletriptan & N02CC06 \\
\hline & Frovatriptan & $\mathrm{N} 02 \mathrm{CCO}$ \\
\hline & Ergot alkaloids & \\
\hline & Ergotamine & N02CA52 \\
\hline & Non-migraine specific analgesics & \\
\hline & Paracetamol & N02BE01 \\
\hline & Treo & N02BA51 \\
\hline & Ibuprofen & M01AE01 \\
\hline & Naproxen & M01AE02 \\
\hline & Tolfenamsyre & M01AG02 \\
\hline & Diclofenac & M01AB05 \\
\hline \multirow[t]{14}{*}{ Prophylactic } & Beta-blocker & \\
\hline & Metoprolol & C07AB02 \\
\hline & Propranolol & C07AA05 \\
\hline & Angiotensin II antagonist & \\
\hline & Candesartancilexetil & C09CA06 \\
\hline & ACE Inhibitor & \\
\hline & Lisinopril & CO9AA03 \\
\hline & Antiepileptics & \\
\hline & Topiramate & N03AX11 \\
\hline & Valproate & N03AG01 \\
\hline & Antidepressive & \\
\hline & Amitriptyline & N06AA09 \\
\hline & Others & \\
\hline & Pizotifen & N02CX01 \\
\hline
\end{tabular}

100) of the assessed patients and used the confusion matrix function from the $\mathrm{R}$ package (caret to retrieve specificity, sensitivity and accuracy [11]. To test the influence of age, gender, and the interaction thereof, we used logistic regression to compare the contribution of each covariate towards the treatment effect and the influence on receiver operating characteristics (ROC). The number of purchases is given as median and quartiles as these data are not normally distributed.

\section{Results}

A total of 1913 migraine patients were questioned about the use and effect of acute and prophylactic migraine 
treatments. Treatment effect of ACE-inhibitors and anti-depressive medication were reported by less than $5 \%$ and were only included in the combined analysis of all prophylactic treatments, see Table 2 .

\section{Acute treatment}

Increased number of purchases was significantly associated with a reporting of positive effect of any acute treatment $(p<1 \mathrm{e}-16)$. However, this signal was markedly driven by triptan purchase (Fig. 1), as both ergotamine and non-migraine specific analgesics did not differ regarding treatment effect $(p>0.05)$. Distribution of ergotamine and non-migraine specific analgesic acute treatments is presented in Additional file 1: Figure S1. Using logistic regression, we included age and gender but did not find a significant contribution to the ROCcurve, see Additional file 1: Figure S5. We calculated the sensitivity, specificity and accuracy at different thresholds for the number of purchases of triptans for prediction of a positive treatment response using a simple confusion matrix (Fig. 2). The overall sensitivity, i.e. the ability to identify a true positive response (Fig. 2 - green line), was above $80 \%$ at all triptan purchase thresholds analysed. However, the specificity, i.e. the ability to detect a true negative response, depended more on the number of purchases. Here, at least ten purchases were needed to gain $70 \%$ specificity (Fig. 2 - blue line). Here, $73 \%$ of the cohort purchased at least ten triptans (Fig. 1). The accuracy, i.e. the ability to predict overall true findings, was above $80 \%$ at all thresholds of purchases analysed. As triptans are known to have higher efficacy in migraine without aura than in migraine with aura, we repeated the analysis excluding migraine with aura patients [12]. Although reducing the statistical power, we found a generally better sensitivity but a lower specificity for migraine without aura, see Additional file 1: Figure S3.

In Denmark, it is clinical practice for neurologists to try at least three different triptans before concluding that triptans do not have an effect (personal communication prof. Jes Olesen). Thus, we tested whether having tried four or more different triptans could indicate a negative treatment response. We calculated a sensitivity of $21 \%$ and a specificity of $73 \%$ to predict a negative treatment response with an accuracy of $63 \%$, see the distribution in Additional file 1: Figure S2. Furthermore, using the number of purchases we tested whether having only one prescription could predict negative treatment response. We found a sensitivity of $80 \%$ and a specificity of $6 \%$ with an accuracy of $20 \%$. For the most prescribed drug, sumatriptan, we also analysed whether patients in monotherapy $(n=50)$ had different purchase number with a sensitivity of $80 \%$. Noting that the number of patients in monotherapy was small, we observed that fewer, six purchases, gave the same results as for all triptans combined.

\section{Prophylactic treatment}

We identified an association between the number of purchases of prophylactic drug and a positive treatment effect $(p<1 \mathrm{e} 16)$, see Fig. 3 . The results did not change when excluding patients with comorbid epilepsy $(n=85)$ or hypertension $(n=473)$, although the average number of purchases dropped significantly for antiepileptics, see Additional file 1: Figure S4. To obtain a specificity above $50 \%$, at least three purchases of angiotensin II antagonist and antiepileptics and four purchases for beta-blockers was needed (Fig. 4 - blue line). We observed that 68, 63, and $63 \%$ of the sample had purchased angiotensin II antagonist and antiepileptic three times and beta-blockers four times. The sensitivity is relatively high $(>80 \%)$ for four purchases or less.

\section{Discussion}

Using a large clinical migraine cohort $(n=1913)$ including information on treatment response, we found a highly significant association between the number of

Table 2 Number of patients with or without an effect of migraine medication

\begin{tabular}{|c|c|c|c|c|c|c|c|}
\hline & \multirow[b]{3}{*}{ Drug category } & \multicolumn{6}{|c|}{ Treatment } \\
\hline & & \multicolumn{2}{|c|}{ All $(n=1913)$} & \multicolumn{2}{|c|}{ Males $(n=540)$} & \multicolumn{2}{|c|}{ Females $(n=1373)$} \\
\hline & & Yes & No & Yes & No & Yes & No \\
\hline \multirow[t]{3}{*}{ Acute } & Triptans & 1113 & 252 & 252 & 65 & 861 & 187 \\
\hline & Non-migraine specific analgesics & 482 & 1080 & 159 & 288 & 323 & 792 \\
\hline & Ergotamine & 89 & 132 & 23 & 21 & 66 & 111 \\
\hline \multirow[t]{5}{*}{ Prophylactic } & Beta-blocker & 187 & 486 & 33 & 112 & 154 & 374 \\
\hline & Angiotensin II antagonists & 232 & 325 & 47 & 73 & 185 & 252 \\
\hline & Antiepileptic & 120 & 325 & 28 & 69 & 92 & 256 \\
\hline & ACE-inhibitor ${ }^{a}$ & 1 & 21 & 1 & 14 & - & 7 \\
\hline & Anti-depressive ${ }^{a}$ & 3 & 27 & 3 & 17 & - & 10 \\
\hline
\end{tabular}




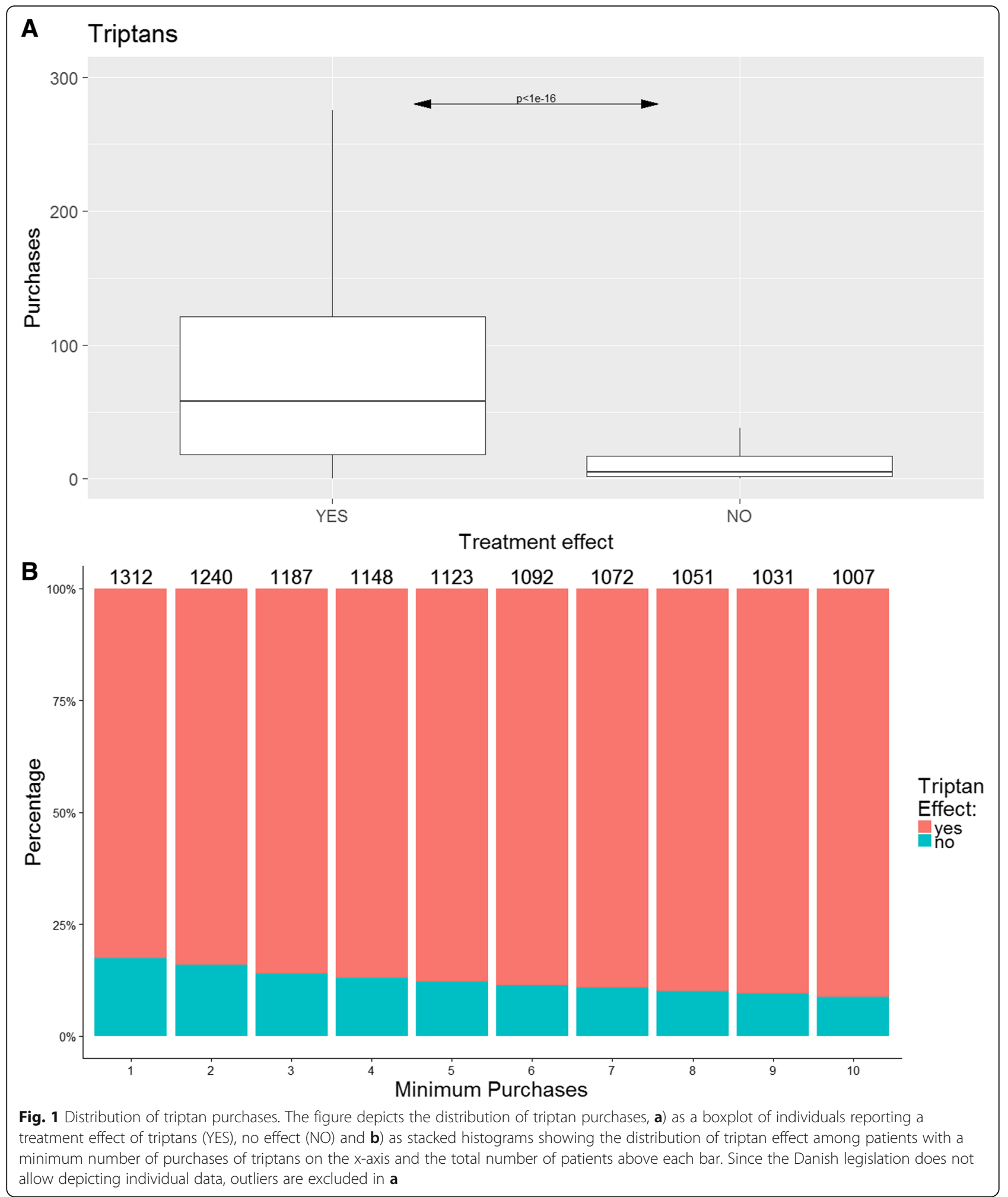

purchases of triptans and positive treatment response, defined as having at least $50 \%$ reduction in symptoms within two hours of treatment start. We showed that the sensitivity was high $(>80 \%)$ for prediction of positive response to triptans and that the sensitivity was above $70 \%$ if the patients had more than ten purchases. We did not find evidence for an association between treatment response and purchases of non-migraine specific 


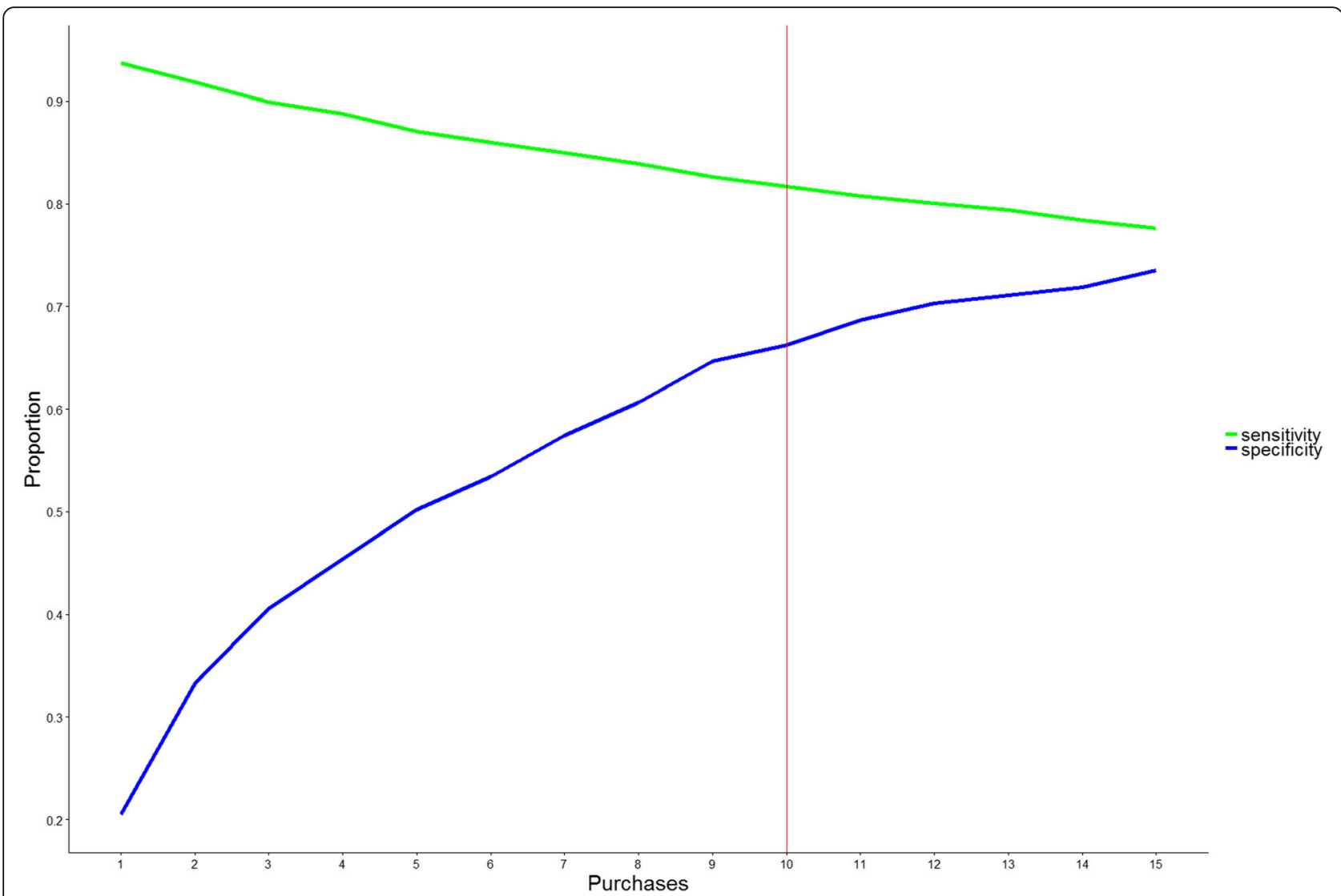

Fig. 2 The sensitivity and specificity for triptan purchases to predict a positive treatment response. The figure presents the sensitivity (green) and specificity (blue) at different thresholds of minimum number of triptan-purchases, e.g. patients with ten or more triptan purchases had a sensitivity of $82 \%$ and specificity of $66 \%$ for prediction of positive treatment response (red line)

analgesics or ergotamines. We did not expect to observe an association, as non-migraine specific analgesics can be bought over-the-counter and are used for many other indications putatively introducing substantial statistical noise.

It is expected that migraine patients who purchase triptans more than once only do so if they experience a positive response. However, we observed an expected increase in sensitivity with number of purchases, although ten purchases of triptans were needed to reach $70 \%$ sensitivity. Since this is a retrospective study, this may be a consequence of recall bias. In addition, the positive response criterion of having $50 \%$ reduction of symptoms within two hours might exclude patients with marginally less or slower effect of triptans. An interesting future study would be to assess the individual migraine drugs. We demonstrated that analysing single drugs necessitates fewer purchases of the drug to gain the $80 \%$ specificity. However, a larger sample size is needed and, although more labour intensive, prospective studies may be needed. However, a key future goal is to compare treatment outcome with genetics, which requires that all participants must be genotyped. Here, thousands of patients are usually required to gain enough statistical power to assess common variants. Thus, a prospective migraine diary-based study seems less feasible.

We found a highly significant association between purchases of prophylactic drugs and a positive treatment response, defined as a more than a 50\% reduction of their migraine attacks, from using angiotensin II antagonists, beta-blockers, or antiepileptics. We found a high sensitivity $(>80 \%)$ predicting positive treatment response from the number of purchases, and a specificity above $70 \%$ after at least three or four purchases depending on the drug. Whereas triptans are specific migraine drugs, the prophylactic drugs are not. As a result, the prediction model for prophylactic drugs necessitates knowledge of the prescription indication. Some drugs may be used to treat a multitude of conditions (e.g. epilepsy, arterial hypertension). Hence, we repeated the analysis excluding patients with comorbid disease. The association remained significant, 

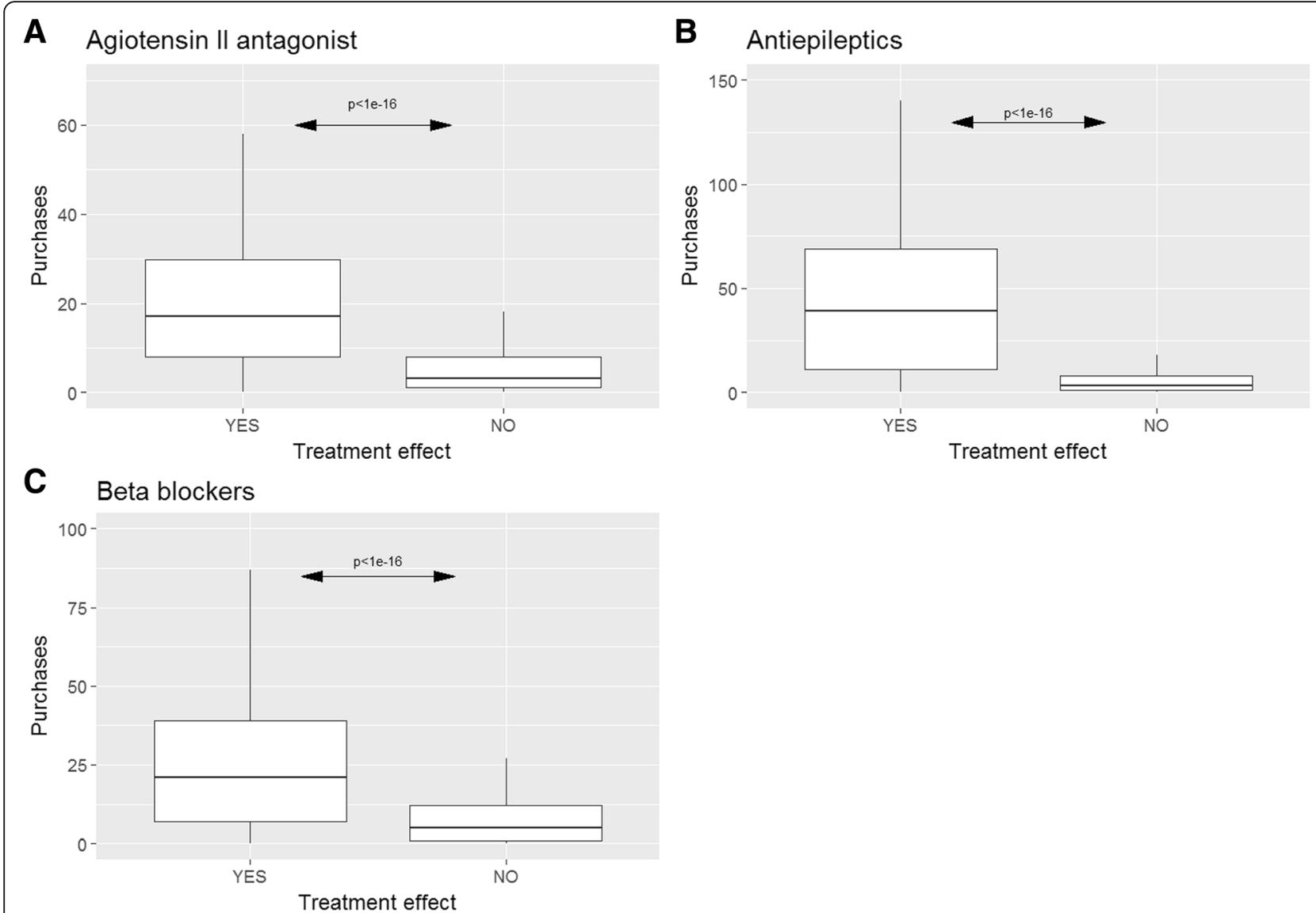

Fig. 3 Distribution of purchases of prophylactic treatment. The figure depicts the distribution of a) Angiotensin II antagonist, b) Antiepileptic, and c) Beta-blockers prescription as a boxplot of patients reporting a treatment effect of triptans (Yes), or no effect (No). Given the Danish legislation it is not allowed to depict individual data, thus outliers are excluded

although we observed a significant drop in the average number of purchases of antiepileptics.

Our study design included general triptan use and response, thus we were unable to test for an association between number of different triptans purchased and patients reporting no treatment effect. Future studies including more complex analysis of the types of triptans, patterns of medication exposures, etc., may aid in the characterization of migraine patient groups.

Precision medicine may offer new strategies to treat migraine, and access to existing large cohorts and pharmacy databases may help reach the required larger sample sizes. Based on the current results we recommend using ten purchases for triptans and three or four purchases for prophylactics. We anticipate that using fewer purchases, e.g. three triptans, may be sufficient; however, this should be evaluated in future studies. Notably, more than $75 \%$ of the triptan users have purchased triptans at least ten times, and 55-
$63 \%$ of the prophylactic users have at least three or four purchases. Additional factors may also influence treatment response, such as the use of other drugs [13-15], i.e. polypharmacy, as well as presence of co-morbid disorders. Here, it is possible that temporal aspect when additional drugs are prescribed, as a prescription could reflect treatment of adverse-effects. Further, it is easy to imagine that migraine patients with co-morbid depression may experience a better response to anti-depressants [16]. Most likely, genetic factors may also condition the treatment response, as seen for lithium and anti-psychotics [17-19], although studies on migraine drugs so far have been inconclusive or lack replication [13, 15].

\section{Conclusions}

We conclude that a national pharmacy database is a valuable resource to identify a positive treatment response for migraineurs. As a general recommendation, we suggest using ten purchases for triptans and 


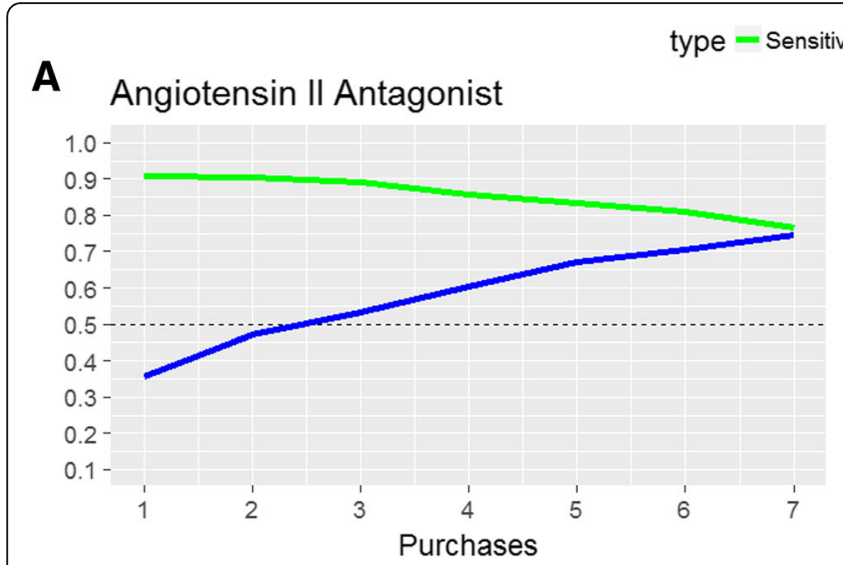

\section{B \\ Antiepileptic}

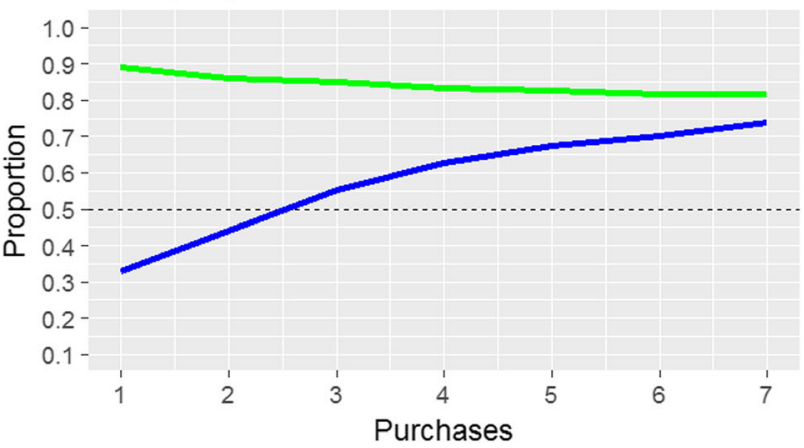

\section{Beta blockers}

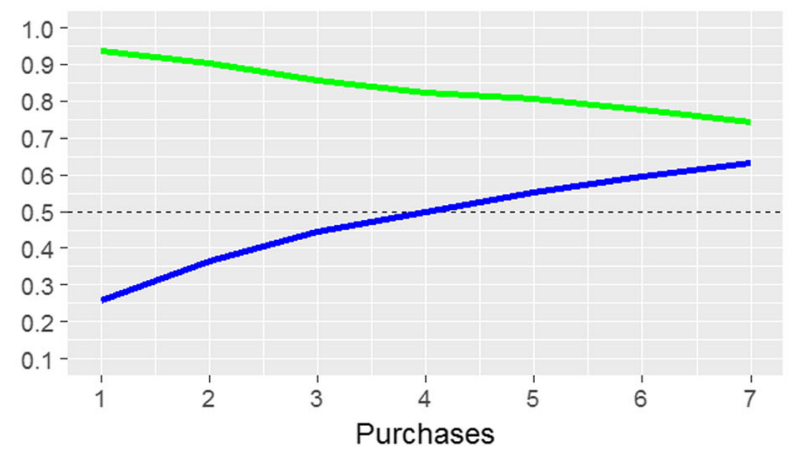

Fig. 4 The sensitivity, specificity, and accuracy of predicting positive treatment response for prophylactic treatment. The figure presents the sensitivity (green), and specificity (blue) at different thresholds of minimum number of purchases to predict a positive treatment outcome. Letters referrers to the three different prophylactic treatments most commonly used, a Angiontensin II Antagonist, b Antiepileptic, \& c Beta blockers

three or four purchases for prophylactic drugs, to predict positive treatment response. In future studies, more detailed information about treatment response and failure would improve the correlation with the number of purchases and perhaps enable us to predict treatment failure. We expect that a lower number of purchases than the ten suggested here may be sufficient.

\section{Additional file}

Additional file 1: Figure S1. Distribution of prescription drugs for total acute treatment, Ergotamine and weak analgesic. Figure S2. Distribution of purchases given the number of different triptans purchased. Figure S3. Distribution and prediction for purchases for migraine patients without aura only. Figure S4. Distribution of prophylactic drugs with and without comorbid disoders. Figure S5. Receiver operating characteristic curve for model with and without gender and age as covariates. Table S1. Questions from semi structured interview. (DOCX $110 \mathrm{~kb}$ )

\section{Abbreviations}

ACE: Angiotensin-converting-enzyme; ICHD: International Classification of Headache Disorders; OTC: Over-the-counter. This referrers to drugs that can be bought without a prescription; ROC: Receiver operating characteristics

\section{Acknowledgements}

We wish to express a special thanks to the research participants of the migraine study.

Funding

CANDYS Foundation (CEHEAD).

\section{Availability of data and materials}

All data generated or analysed during this study are included in this published article (and its Additional file).

\section{Authors' contributions}

TFH and JO: Wrote the paper. TFH, TMH, MAC, and LK, conceived and designed the experiment. TFH analysed and interpreted the data. TFH, MAC, JO contributed reagents, materials, analysis tools or data. All authors applied critical review of manuscript and subsequent approval.

\section{Ethics approval and consent to participate}

Written informed consent was obtained from all participants. The study was approved by the Danish Ethical Scientific Committee (H-2-2010-122) and the Danish Data Protection Agency (01080/GLO-2010-10). The obtained approval from the Ethical Scientific Committee and Data Protection Agency states that data are not allowed in any personal identifiable form, and only summarized statistics can be shared.

\section{Consent for publication}

This publication does not include individualized data.

\section{Competing interests}

The authors declare that they have no competing interests. 


\section{Publisher's Note}

Springer Nature remains neutral with regard to jurisdictional claims in published maps and institutional affiliations.

\section{Author details}

'Danish Headache Centre, Department of Neurological department, Copenhagen University Hospital, Nordreringvej 69, DK-2600 Glostrup, Denmark. ${ }^{2}$ Novo Nordisk Foundation Center for Protein Research, Faculty of Health and Medical Sciences, University of Copenhagen, Copenhagen, Denmark. ${ }^{3}$ Institute of Biological Psychiatry, Mental Health Centre Sct. Hans, Copenhagen University Hospital, Roskilde, Denmark.

Received: 29 November 2018 Accepted: 20 March 2019 Published online: 02 April 2019

\section{References}

1. Lee YH, Bang H, Kim DJ (2016) How to establish clinical prediction models. Endocrinol Metab (Seoul) 31(1):38-44

2. Garcia Rodriguez LA, Perez Gutthann S (1998) Use of the UK general practice research database for pharmacoepidemiology. Br J Clin Pharmaco 45(5):419-425

3. Stovner LJ, Zwart JA, Hagen K, Terwindt G, Pascual J (2006) Epidemiology of headache in Europe. Eur J Neurol 13(4):333-345

4. Antonaci F, Dumitrache C, Cillis I, Allena M (2010) A review of current European treatment guidelines for migraine. J Headache Pain 11(1):13

5. Tepper SJ, Spears RC (2009) Acute treatment of migraine. Neurol Clin 27(2):417-427

6. Loder E (2010) Triptan therapy in migraine. N Engl J Med 363(1):63-70

7. Ramadan NM (2007) Current trends in migraine prophylaxis. Headache 47 Suppl 1:S52-7

8. Kildemoes HW, Sorensen HT, Hallas J (2011) The Danish National Prescription Registry. Scand J Public Health 39(7 Suppl):38-41

9. Esserlind A, Christensen A, Steinberg S, Grarup N, Pedersen O, Hansen T et al (2016) The association between candidate migraine susceptibility loci and severe migraine phenotype in a clinical sample. Cephalalgia 36(7):615

10. Olesen J (2006) International classification of headache disorders, (ICHD-2): current status and future revisions. SAGE Publications Sage UK, London, England

11. Kuhn M (2008) Building predictive models in R using the caret package. J Stat Softw 28(5):1-26

12. Bates D, Ashford E, Dawson R, Ensink FB, Gilhus NE, Olesen J et al (1994) Subcutaneous sumatriptan during the migraine aura. Sumatriptan Aura study group. Neurology. 44(9):1587-1592

13. Zhang LM, Dong Z, Yu SY (2016) Migraine in the era of precision medicine. Ann Transl Med 4(6):105

14. Capi M, Gentile G, Lionetto L, Salerno G, Cipolla F, Curto M et al (2018) Pharmacogenetic considerations for migraine therapies. Expert Opin Drug Metab Toxicol:14(11):1161-14(11):1167

15. Kojic Z, Stojanovic D (2013) Pathophysiology of migraine--from molecular to personalized medicine. Med Pregl 66(1-2):53-57

16. Pomes LM, Gentile G, Simmaco M, Borro M, Martelletti P (2018) Tailoring treatment in Polymorbid migraine patients through personalized medicine. CNS Drugs 32(6):559-565

17. Ingason A, Rujescu D, Cichon S, Sigurdsson E, Sigmundsson T, Pietilainen $\mathrm{OPH}$ et al (2011) Copy number variations of chromosome 16p13.1 region associated with schizophrenia. Mol Psychiatry 16(1):17-25

18. Ravyn D, Ravyn V, Lowney R, Nasrallah HA (2013) CYP450 pharmacogenetic treatment strategies for antipsychotics: a review of the evidence. Schizophr Res 149(1-3):1-14

19. Ingelman-Sundberg M (2004) Pharmacogenetics of cytochrome P450 and its applications in drug therapy: the past, present and future. Trends Pharmacol Sci 25(4):193-200

Ready to submit your research? Choose BMC and benefit from:

- fast, convenient online submission

- thorough peer review by experienced researchers in your field

- rapid publication on acceptance

- support for research data, including large and complex data types

- gold Open Access which fosters wider collaboration and increased citations

- maximum visibility for your research: over $100 \mathrm{M}$ website views per year

At $\mathrm{BMC}$, research is always in progress.

Learn more biomedcentral.com/submissions 